\title{
Estudos Sobre a Cinética de Decomposição Térmica do Poli(Sulfeto de Fenileno)
}

\author{
Laércio G. Lage e Yoshio Kawano
}

Resumo: A cinética de decomposição térmica do poli(sulfeto de fenileno) (PPS) foi estudada através da termogravimetria (TG). Duas amostras comerciais de PPS foram utilizadas neste trabalho: Ryton, da Philips Petroleum, e Fortron, da Hoechst-Celanese. Os parâmetros cinéticos, isto é, a energia de ativação e o fator pré-exponencial de Arrhenius, foram calculados através de três métodos diferentes. Os resultados aqui apresentados concordaram com os dados da literatura. A estimativa do tempo de meiavida do polímero também foi feita, mostrando que o Fortron é mais estável termicamente que o Ryton.

Palavras-chave: Poli(sulfeto de fenileno), termogravimetria, cinética, energia de ativação, tempo de meia-vida.

\section{Introdução}

O poli(sulfeto de fenileno) (PPS) é um homopolímero linear, termoplástico e semi-cristalino ${ }^{[1-2]}$. A sua fórmula estrutural está representada na Figura 1.

O PPS possui boa estabilidade térmica, alta resistência química, baixa absorção de água e boas propriedades mecânicas. Alguns estudos tem sido feitos na tentativa de melhorar suas propriedades mecânicas ${ }^{[3-4]}$, bem como na tentativa de torná-lo um polímero condutor ${ }^{[5-6]}$. Atualmente, o seu emprego vai desde o setor eletro-eletrônico, passando pelo automobilístico, de eletrodomésticos, petroquímico e de utensílios de cozinha.

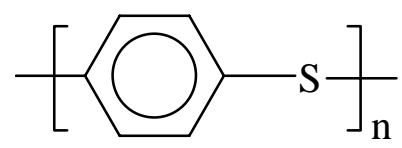

Figura 1. Fórmula estrutural do PPS.
Uma das crescentes aplicações do PPS no Brasil se dá na área de tintas anti-aderentes à base de água, a fim de empregá-las em utensílios de cozinha. Para a fabricação de tintas anti-aderentes utilizam-se resinas de poli(amida-imida) (PAI), poli(etileno sulfona) (PES) ou PPS, todas em conjunto com politetrafluoroetileno (PTFE). As tintas formuladas com PES ou PAI possuem uma resistência mecânica superior às baseadas em PPS; todavia, as formuladas com PPS possuem uma maior resistência térmica, além de não utilizarem solventes orgânicos para sua dispersão ${ }^{[1]}$.

Devido aos usos e propriedades do PPS, temse aumentado o interesse no conhecimento do seu comportamento em condições extremas ${ }^{[2,7-8]}$. O estudo cinético de decomposição térmica de materiais através da termogravimetria (TG) tem sido uma ferramenta útil para a estimativa do tempo de meiavida do polímero em determinadas condições, bem como calcular os parâmetros cinéticos envolvidos 
no processo ${ }^{[9-10]}$. Alguns fatores são determinantes para o cálculo destes parâmetros, como as condições experimentais (massa da amostra, tipo de cadinho, razão de aquecimento e atmosfera) e o método de cálculo empregado. A necessidade de padronizar tais fatores levou a ASTM a propor uma norma (ASTM E 1641-94) para o estudo cinético através de $\mathrm{TG}^{[11]}$. Em vista dos parâmetros calculados serem dependentes do método de cálculo, optou-se neste trabalho a utilização de 3 métodos diferentes: o método proposto pela ASTM (método 1), o método disponível no "software" do aparelho de $\mathrm{TG}^{[12]}$ (método 2) e o método de Kissinger ${ }^{[13]}$ (método 3 ).

Os métodos 1 e 2 possuem o mesmo princípio: o cálculo da energia de ativação em diferentes graus de conversão da amostra, com diferentes razões de aquecimento. Ambos são métodos integrais, baseados no método de Ozawa-FlynnWall ${ }^{[14-15]}$. A diferença entre eles é que o método 1 foi simplificado a fim de poder ser empregado com cálculos facilmente executáveis, enquanto que o segundo requer o uso do programa computacional. Uma limitação do método 1 é a de ser aplicável em amostras que possuam uma única etapa de decomposição e cuja cinética deva ser de primeira ordem. Esta última limitação também faz parte do método 2 .

O método 3 é um método diferencial e a energia de ativação é calculada no ponto onde a taxa de reação é máxima, com diferentes razões de aquecimento. Este método possui a vantagem de não ter limitação quanto ao número de etapas, além de ser possível calcular também a ordem de reação.

As equações que descrevem cada método são as seguintes:

-Para o método 1 (ASTM) ${ }^{11}$ :

$$
\begin{aligned}
& \log q=-\left(E_{e} b / R\right)(1 / T) \\
& A=-\ln (1-\alpha) 10^{a} /\left(E_{r} / R^{\prime}{ }^{\prime}\right)
\end{aligned}
$$

onde q é a razão de aquecimento; $\mathrm{E}_{\mathrm{e}}$ a energia de ativação estimada; $b$ uma variável de iteração; $\mathrm{R}$ a constante dos gases, $\mathrm{T}$ a temperatura absoluta, A o fator pré-exponencial de Arrhenius, $\alpha$ o grau de conversão, a o valor aproximado de integração, $E_{r}$ o valor convergido de energia de ativação e q' o valor médio entre as razões de aquecimento empregadas. $\mathrm{O}$ cálculo de $\mathrm{E}_{\mathrm{e}}$ é feito para cada grau de conversão e é repetido diversas vezes, mudando o valor de $b$, até atingir uma diferença menor de $1 \%$ entre os resultados, onde passa a chamarse $E_{r}$, calculando-se então o valor de A.

-Para o método 2 (programa TG) ${ }^{[12]}$ :

$\log \mathrm{q} \approx \log \left\{\mathrm{AE}_{\mathrm{a}} / \mathrm{R} \int[\mathrm{d} \alpha / \mathrm{f}(\alpha)]\right\}+2,315-(0,457 / \mathrm{R}) \mathrm{E}_{\mathrm{a}}(1 / \mathrm{T})$

onde $E_{a}$ é a energia de ativação. $O$ cálculo é feito para cada grau de conversão.

Para o método 3 (Kissinger) ${ }^{[13]}$ :

$\ln \left(\mathrm{q}_{\max } / \mathrm{T}_{\max }{ }^{2}\right)=\left(-\mathrm{E}_{\mathrm{a}} / \mathrm{R}\right)\left(1 / \mathrm{T}_{\max }\right)+\ln \left[\left(\mathrm{AR} / \mathrm{E}_{\mathrm{a}}\right) \mathrm{n}(1-\alpha)^{\mathrm{n}-1}\right]$

onde $\mathrm{n}$ é a ordem de reação e o sub-índice max indica que os valores são tomados onde a taxa de reação é máxima.

A energia de ativação é proporcional ao coeficiente angular das retas obtidas dos gráficos de log q versus $1 / \mathrm{T}$ (métodos 1 e 2 ) ou de $\ln \left(\mathrm{q}_{\max } / \mathrm{T}_{\max }{ }^{2}\right.$ ) versus $1 / \mathrm{T}_{\max }$ (método 3 ).

Os objetivos deste trabalho foram o de comparar o comportamento e a estabilidade térmica de dois tipos de amostras comerciais de PPS: Ryton, fabricado pela Philips Petroleum, e Fortron, pela Hoechst-Celanese, utilizando a técnica de TG para aquisição de dados, e a comparação de resultados obtidos através de diferentes métodos de cálculo.

\section{Experimental}

Dois tipos de amostras de PPS foram utilizadas neste trabalho: Ryton, que possui grupos terminais $\mathrm{SH}$, e Fortron, com grupos terminais $\mathrm{Cl}$. Para as corridas de TG foi utilizado o aparelho HiRes $^{\text {TM }}$ TGA 2950, da TA Instruments, interfaceado ao "software" Thermal Analyst 2000. As massas iniciais das amostras variaram entre 3 e $4 \mathrm{mg}$. As condições utilizadas no aparelho foram: razões de aquecimento de $1,2,5,7$ e $10^{\circ} \mathrm{C} \cdot \mathrm{min}^{-1}$; cadinho de platina; sensibilidade 1 e atmosfera de nitrogênio, num fluxo constante de $100 \mathrm{~cm}^{3} \cdot \mathrm{min}^{-1}$. As corridas foram feitas de 25 a $650^{\circ} \mathrm{C}$.

\section{Resultados e discussão}

A decomposição térmica do PPS até $650^{\circ} \mathrm{C}$, em atmosfera inerte de nitrogênio, se deu em apenas uma etapa, o que possibilitou a aplicação de 
Tabela 1. Parâmetros cinéticos calculados para a decomposição térmica do PPS.

\begin{tabular}{|c|c|c|c|c|c|c|}
\hline PPS & $\begin{array}{c}\mathrm{E}_{\mathrm{a}}(\text { método 1) / } \\
\left(\mathrm{kJJmol}^{-1}\right)\end{array}$ & 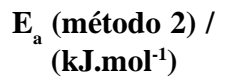 & $\begin{array}{c}\mathrm{E}_{\mathrm{a}}(\text { método 3) / } \\
\left(\mathrm{kJJmol}^{-1}\right)\end{array}$ & $\begin{array}{l}\text { A (método 1) / } \\
\left(10^{10} \text { min }^{-1}\right)\end{array}$ & $\begin{array}{c}\text { A (método 2) / } \\
\left(10^{10} \mathrm{~min}^{-1}\right)\end{array}$ & $\begin{array}{c}\text { A (método 3) / } \\
\left(10^{10} \min ^{-1}\right)\end{array}$ \\
\hline Ryton & 168 & 155 & 168 & 1,47 & 0,258 & 1,82 \\
\hline Fortron & 159 & 150 & 192 & 0,336 & 0,103 & 8,19 \\
\hline
\end{tabular}

todos os métodos de cálculo escolhidos. Os gráficos utilizados para os cálculos de energia de ativação estão dados nas Figuras 2 e 3 . A Tabela 1 mostra os parâmetros cinéticos calculados pelos diferentes métodos.

Da comparação destes valores, nota-se que o método 2 é o que produz os menores valores de energia de ativação e, conseqüentemente, menores valores do fator pré-exponencial. Day et al ${ }^{[9]}$. estudaram a cinética de decomposição térmica do PPS Ryton e Fortron e, utilizando o método de Kissinger, obtiveram os valores de energia de ativação de $162 \mathrm{~kJ} \mathrm{~mol}{ }^{-1}$ para o Ryton e $178 \mathrm{~kJ} \cdot \mathrm{mol}^{-1}$ para o Fortron. Pelo método de Ozawa-FlynnWall[14-15], os valores obtidos foram $183 \mathrm{~kJ}^{-\mathrm{mol}^{-1}}$ para o Ryton e $169 \mathrm{~kJ}^{\mathrm{m}} \mathrm{mol}^{-1}$ para o Fortron. Os resultados obtidos neste trabalho e os de Day et $\mathrm{al}^{[9]}$. se mostraram concordantes, dentro de um erro máximo de $10 \%$. O cálculo de $\mathrm{A}$ por Day et a $\mathrm{a}^{[9]}$. produziu valores em torno de $10^{14} \mathrm{~min}^{-1}$ para o Ryton e $10^{13} \mathrm{~min}^{-1}$ para o Fortron, pelo método de Ozawa-Flynn-Wall[14-15]. Day et a ${ }^{[9]}$ não calcularam os valores de A pelo método de Kissinger ${ }^{[13]}$. Calcularam A também através de dados de experimentos isotérmicos, utilizando gráficos de taxa de reação e de tempo reduzido e os resultados estavam em torno de $10^{10} \mathrm{~min}^{-1}$ para o Ryton, por ambos os gráficos, e em torno de $10^{10} \mathrm{e} 10^{8} \mathrm{~min}^{-1}$

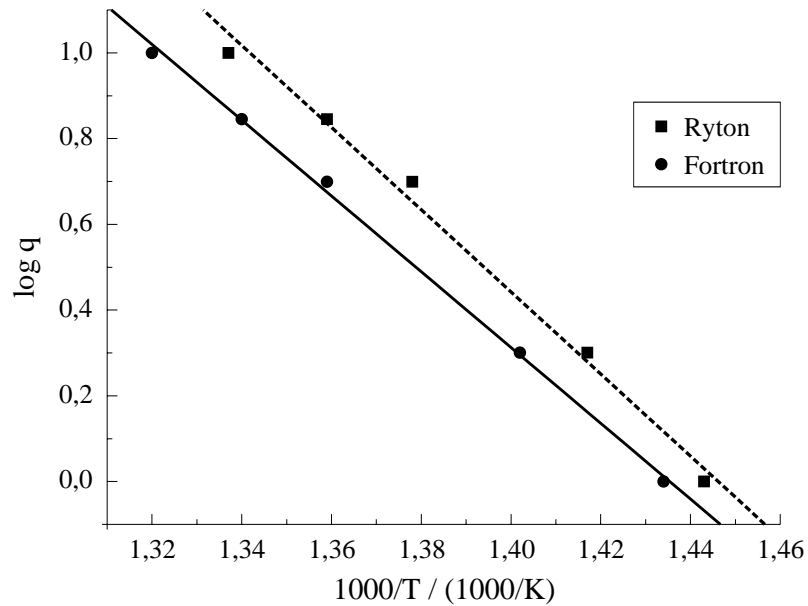

Figura 2. Gráfico de $\log$ q vs. 1000/T para grau de conversão de $5 \%$, utilizado nos métodos 1 e 2 . para o Fortron, pelo gráfico de taxa de reação e de tempo reduzido, respectivamente, produzindo valores mais próximos aos calculados neste trabalho. A energia de ativação calculada pelos gráficos de taxa de reação e de tempo reduzido foram, respectivamente, 166 e $180 \mathrm{~kJ}^{\mathrm{mol}}{ }^{-1}$ para o Ryton e 145 e $148 \mathrm{~kJ} . m o l^{-1}$ para o Fortron.

$\mathrm{O}$ método 3 possibilitou o cálculo da ordem de reação $\mathrm{n}$ do polímero, que situou-se na faixa de 1 para ambos os tipos de PPS (reação de $1^{\text {a }}$ ordem), o que valida a aplicação dos métodos $1 \mathrm{e}$ 2. Segundo Denq et a ${ }^{[16]}$. reações de $1^{\mathrm{a}}$ ordem em polímeros estão relacionadas a quebras aleatórias de ligações da cadeia principal e transferência intermolecular. Estudos da decomposição do PPS realizados por Budgell et a ${ }^{[2]}$. utilizando a técnica de pirólise acoplada à cromatografia gasosa e espectrometria de massa, demonstraram que as reações que ocorriam nas condições de temperatura semelhantes as dos experimentos realizados neste trabalho foram interpretadas como sendo de primeira ordem global, reforçando a validade de aplicação dos métodos 1 e 2 . Nestes estudos de Budgell et al ${ }^{[2]}$. foram identificados, até $550^{\circ} \mathrm{C}$, a presença de tetrâmeros cíclicos e dímeros e trímeros lineares. Na faixa de 550 a $650^{\circ} \mathrm{C}$, ocorre o aparecimento de benzotiofenos e naftotiofenos. O processo de decomposição predominante parece ser

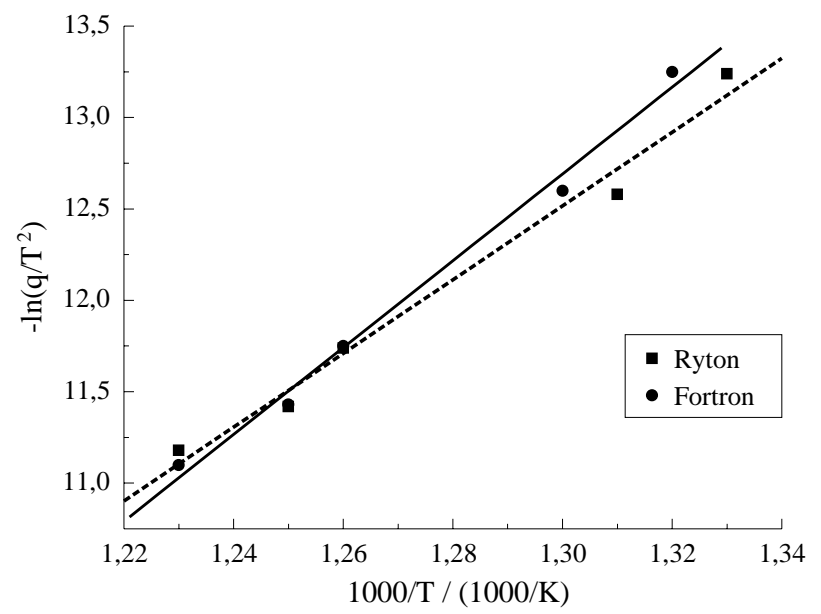

Figura 3. Gráfico de - $\ln \left(\mathrm{q} / \mathrm{T}^{2}\right)$ vs. 1000/T utilizado no método 3. 
Tabela 2. Tempos de meia-vida do PPS em diferentes temperaturas.

\begin{tabular}{cccc}
\hline PPS & $\mathbf{t}_{\mathbf{4 0 0}{ }^{\circ} \mathrm{C}} /(\mathbf{m i n})$ & $\mathbf{t}_{\mathbf{5 0 0}{ }^{\circ} \mathbf{C}} /(\mathbf{m i n})$ & $\mathbf{t}_{\mathbf{6 0 0}^{\circ} \mathbf{C}} /(\mathbf{m i n})$ \\
\hline Ryton & 396 & 9,38 & 0,524 \\
Fortron & 510 & 20,3 & 1,69 \\
\hline
\end{tabular}

a cisão aleatória de ligações das cadeias do polímero, seguida de ciclização em temperaturas até $550^{\circ} \mathrm{C}$ e depolimerização em temperaturas superiores. Segundo Denq et al., ${ }^{[16]}$ as reações de ciclização e depolimerização são reações de ordem zero; sendo assim, a ordem global de reação é 1, o que confirma os resultados calculados pelo método 3 .

O tempo de meia-vida estimado para os polímeros foi escolhido como aquele em que há aproximadamente $5 \%$ de perda de massa do material, em diferentes temperaturas, conforme ilustrado na Tabela 2. O cálculo foi feito utilizando-se apenas o método 2 .

Observando-se os dados da Tabela 2, nota-se que a estabilidade térmica do PPS Fortron é pouco maior que a do PPS Ryton, dada pelo tempo de meia-vida dos polímeros. Alguns autores creditam esta diferença de estabilidade devido aos grupos terminais de cada polímero ( $\mathrm{Cl}$ no caso do Fortron e SH no Ryton).

Uma das limitações da estimativa do tempo de meia-vida de materiais é que muitos deles sofrem perda de propriedades físicas de interesse antes de perder massa. Este cálculo é usado apenas como estimativa e comparativo de estabilidade térmica.

\section{Conclusões}

O cálculo dos parâmetros cinéticos da decomposição térmica do PPS permitiu a comparação da estabilidade térmica de dois tipos de PPS comerciais. Notou-se que o PPS Fortron é mais estável termicamente que o PPS Ryton, em condições de atmosfera inerte. Tal estimativa é uma informação útil para avaliar as condições ao qual o material pode ser submetido.

A aplicação de diferentes métodos de cálculo de parâmetros cinéticos demonstrou-se válida para este sistema de estudo, dada a decomposição do PPS se dar em uma etapa e possuir ordem de reação igual a um. Para este sistema, os parâmetros cinéticos tiveram valores concordantes entre si e com os reportados na literatura, mesmo utilizando diferentes métodos de cálculo e condições experimentais diversas.

Os valores de tempo de meia-vida mostraram que o PPS é um material termoplástico durável.

\section{Agradecimentos:}

À Adriana Marçula pelo trabalho de caracterização do PPS e pela doação de amostras; à FAPESP e ao CNPq pelo auxílio financeiro.

\section{Referências Biliográficas:}

1. Marçula, A. - Dissertação de Mestrado, Instituto de Química da Universidade de São Paulo;

2. Budgell, D.R.; Day, M.; Cooney, J.D. - Polym. Degr. Stab. 43, 109, (1994).

3. D’llario, L.; Martinell, A.; Piozzi, A. - Eur. Polym. J. 28, 643, (1992).

4. Bohning, M.; Schultze, J.D.; Springer, J. - Macromol. Chem. Phys. 195, 2579, (1994).

5. Yang, Y.; D’Amore, A.; Di, Y.; Nicolau, L.; Li, B. J. Appl. Polym. Sci. 59, 1159, (1996).

6. Gabellini, G.; Moraes, M. B.; Bretas, R. E. S. - J. Appl. Polym. Sci. 60, 21, (1997).

7. Peters, O. A.; Still, R. H. - Polym. Degr. Stab. 42, 41, (1993).

8. Peters, O A.; Still, R.H. - Polym. Degr. Stab. 42, 49, (1993).

9. Day, M.; Budgell, D. R. - Thermochim. Acta 203, 465, (1992).

10. Lage, L.G.; Kawano, Y. - An. Assoc. Bras. Quím. 47, 321, (1998).

11. Norma ASTM E 1641-94.

12. Manual TGA 2950, TA Instruments, New Castle, EUA, (1994).

13. Kissinger, H. E. - Anal. Chem. 29, 1702, (1957).

14. Ozawa, T. - Bull. Chem. Soc. Jpn. 38, 1881, (1965).

15. Flynn, J. H.; Wall, L. A. - J. Res. NBS - Phys. and Chem. Ed. 70 A, 487, (1966).

16. Denq, B.-L.; Chiu, W.-Y.; Lin, K.-F. - J. Appl. Polym. Sci. 66, 1855, (1997). 